reproduced by any other simple mechanism. Some theorists are hailing this result as the proof that gluons exist, though many people are not fully convinced.

If the upsilon is produced by way of a single photon then it must have negative charge-conjugation parity (Cparity). Since the upsilon is too light to decay to the b-anti-b continuum it can only decay to quarks if the weak interaction turns $\mathrm{a} b$ into a lighter $\mathrm{c}$ quark, but that is a very slow process. In the meantime there is predicted to be a high probability that it can decay into gluons. But gluons are like photons in a number of ways. In particular, they also should have negative C-parity, so the upsilon can only conserve C-parity if it decays to an odd number of gluons. Energymomentum conservation forbids decay to one gluon and the phase-space probability is small for five gluons or more. Sceptics claim, correctly, that other three-jet models can be constructed, but QCD does predict that three gluon jets should be seen and the PLUTO results must therefore be taken seriously as at least circumstantial evidence for the existence of gluons inside hadrons.

\section{Ten years after ... a decade of lunar science}

\section{from C. T. Pillinger}

THE 10th anniversary meeting of the Lunar and Planetary Science Conference* met under the shadow of recent budgetary constraints imposed by the US Congress. The growing 'planetary' nature of the conference was marked; indeed discussion on new data from lunar soils has diminished substantially from its originally dominant position. Data from new planetary missions, meteorites and studies of fundamental processes on the Moon attract more interest from today's planetologist.

A highlight of the conference was the presentation of new results from the Viking, Voyager and Pioneer missions. A short 3-D film of Mars, created from elegant computer techniques by E. Levinthal (Stanford University), preceded spectacular close-up pictures of Jupiter and the Gallilean satellites, providing exciting new venues for geological speculation. The volcanic activity on Io, and apparent tectonic effects on Ganymede, will no doubt provide the basis for much discussion at future meetings. The composition of the atmosphere of Venus, with its apparently 'solar' rare gas abundance (see News \& Views 278, $777 ; 1979$ ), leads one to speculate as to how radically our current ideas of planetary formation may have to be revised over the next 10 years.

As pointed out by S. R. Taylor (Australian National University, Canberra), 10 years study of the Apollo samples has failed to achieve consensus on the origin of the Moon, although considerable constraints can be inferred. Existing disagreements continued over the criteria for estimating siderophile element abundances indigenous to the highland crust and the whole Moon. Delano and Ringwood (Canberra) disputed the elemental correlations calculated by the group of $\mathrm{E}$. Anders (University of Chicago). The importance of the argument is the validity of the assertion by Ringwood that the Moon was derived from the Earth's mantle after formation of the Earth's core, as opposed to condensation as a discrete body. A third position was adopted by Wanke et al. (University of Mainz) who, on the evidence of $\mathrm{Co} /(\mathrm{Fe}+$ $\mathrm{Mg}$ ) ratios, favoured a genetic relationship between the Earth and Moon.

Continued studies of lunar drill core samples have clarified ideas concerning reworking and deposition processes in the lunar surface layer, known in lunar parlance as 'the regolith'. Soil exposure indices have been based on a number of irradiation effects and petrographic particle size data. However, the relative importance of erosion compared with deposition processes (for example sputtering, impact volatilisation and deposition, and micrometeorite erosion) continues to be the subject of lively debate.

Wieler et al. (University of Zurich) showed that 0.5 billion year old soils had similar solar rare gas ratios to the present; no information on the solar wind flux in the past was forthcoming, although various workers have postulated an increased flux of varying isotopic composition in earlier irradiation. The magnitudes of the postulated changes seem to be at variance with existing models of solar processes.

Meteoritic studies have benefited from techniques developed for the lunar programme, and the growth of such investigations was evident. The Allende meteorite in particular has been subjected to intensive study. The Caltech group presented a detailed study of a new hibonite-rich inclusion (HAL); hibonite is considered to be one of the highest temperature major element condensates from the solar nebula. This inclusion consisted of an unusually pure hibonite core and several rims of lower-temperature

*Held at the Johnson Space Center, Houston on 19-23 March. phases. Comparison with other Allende $\mathrm{Ca}, \mathrm{Al}$-rich inclusions implied a large ${ }^{26} \mathrm{Mg}$ anomaly; however, this was not found, suggesting a low ${ }^{26} \mathrm{~A} 1$ content for HAL. Ca isotope shifts in all phases were found to be large but uniform, and small nonlinear effects attributed to a nuclear origin. HAL thus qualifies as a new Allende isotopically-anomalous inclusion. Jessberger et al. (University of Heidelberg) reported a very high ${ }^{39} \mathrm{Ar} /{ }^{40} \mathrm{Ar}$ apparent age of over 5 billion years, which can either be interpreted as a very old component, or a possible ${ }^{40} \mathrm{~K}$ isotopic enhancement. The latter possibility should be resolved by work now under way in Mainz. The existence of very old inclusions ( $>5$ billion years) would imply that either these inclusions originated from outside the condensing solar nebula, or that the currently accepted age of the Solar System of 4.5 billion years needs to be re-assessed.

Strongly nonlinear kinetic isotope fractionations of the three stable isotopes of oxygen have been produced in the laboratory. G. Arrhenius (La Jolla) suggested this effect as another source of the nonlinear isotopic behaviour, to complement the nucleosynthetic processes. The latter were proposed to explain the original discovery of such anomalies in oxygen by R. N. Clayton in 1973.

Recent and previous discoveries of isotopic anomalies place constraints on possible models of the origin of the Solar System. However, considerable latitude is still available, as a number of apparently contradictory models were discussed at length mainly by $\mathrm{D}$. D. Clayton (Rice University) and A. Cameron (Harvard University).

An interesting new sample collection method, which may be important to future research, is the 'cosmic muckrake' of Brownlee and others. Using a magnetic rake dragged across the ocean floor, large quantities of extraterrestrial material can be recovered. In a typical day's operation, the device processed $\sim 10^{7} \mathrm{~kg}$ of sediment, and separated about half a million particles, many of which were identified as extraterrestrial. Debris from nearly every type of object which has collided with Earth over the past $10^{5}$ years might be collected.

The few contributions mentioned outline some promising research emerging in planetary science. The emphasis on the Moon a decade ago has given new impetus to the older science of meteoritics; additionally, new information from hitherto inaccessible objects is now becoming available.

C. T. Pillinger is in the Planetary Science Unit, Department of Mineralogy and Petrology, University of Cambridge. 